\title{
千岛湖岛屿化对社鼠的肥满度之影响
}

\author{
孙波, 鲍毅新 ${ }^{*}$, 张龙龙, 赵庆洋, 胡知渊 \\ （浙江师范大学 生态研究所, 浙江 金华 321004)
}

\begin{abstract}
摘要: 2008 年 5 月以夹夜法对千岛湖区部分岛屿开展小型兽类种群数量调查, 对其间捕获的优势鼠种一一社 鼠 (Niviventer confucianus) 进行解剖观察以及相应的测量, 计算其肥满度指标 $K$ 值 $\left(K=100 \mathrm{~W} / \mathrm{L}^{3}\right)$; 同时对岛屿化 环境下, 社鼠的肥满度与性别、年龄、生境类型、岛屿面积和捕获率等生态因子的关系进行了研究。结果显示: 岛屿化对社鼠两性之间的肥满度产生影响, 其差异程度由大型岛屿的显著差异 $(P=0.015)$ 至中小型岛屿的无显

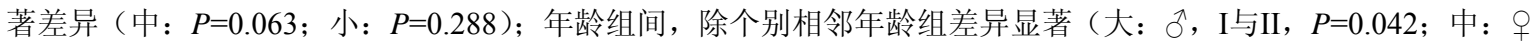
和亏皆为 II 与 III, $P=0.013$ 和 $P=0.044$ ) 或极显著（大: + , III与IV, $P=0.000$ ) 外, 其余均未达到显著水平 $(P>0.05$ )。 社鼠肥满度年龄组间的变化主要与其自身生理特征变化有关, 与岛屿化关系不明显; 岛屿的隔离作用, 致使不同 类型岛屿上的植被优势种及丰富度存在明显差异, 从而影响到社鼠的肥满度差异; 各岛捕获率所示的社鼠种群的 相对数量与其肥满度并无显著相关性 (Pearson-test, $\rho, r=-0.226, P=0.437 ; \hat{O}, r=-0.019, P=0.949$ ), 岛屿化对 其种群数量不产生影响; 不同面积岛屿之间社鼠的肥满度差异极显著 $(P=0.000)$, 且肥满度均值与岛屿面积呈显 著正相关 (Pearson test, $r=0.561, P=0.037$ ), 岛屿面积不同, 对社鼠的肥满度之变化产生影响。总之, 千岛湖岛 屿社鼠的肥满度差异的产生, 除了性别、年龄等因素外, 与岛屿的面积、生境及种群压力有直接或间接的关系, 从侧面也反映了社鼠对岛屿环境的适应性。
\end{abstract}

关键词: 社鼠; 肥满度; 生境片段化; 千岛湖岛屿

中图分类号: Q142.9; Q958.1; Q959.837 文献标识码: A 文章编号：0254-5853(2009)05-0545-08

\section{Preliminary Study on Relative Fatness of Niviventer confucianus on Islands of Qiandao Lake Region}

\author{
SUN Bo, BAO Yi-xin ", ZHANG Long-long, ZHAO Qing-yang, HU Zhi-yuan \\ (Institute of Ecology, Zhejiang Normal University, Jinhua 321004, China)
}

\begin{abstract}
Relative fatness is an important general indicator of physiological body condition in small mammals and reflects their adaptive ability to the environment. In May 2008, we surveyed part islands in Qiandao Lake about the number of populations of small mammals through the night trapping method, anatomizing and measuring the dominant species Niviventer confucianus captured during the search, calculating the index of relative fatness $K,\left(K=100 \mathrm{~W} / \mathrm{L}^{3}\right)$, where $\mathrm{L}$ indicated the body length, and $\mathrm{W}$ was the carcass body weight; Meanwhile we investigates the relationship between the relative fatness and the changing characteristics in sex, age, habitat types and other factors under islanding conditions. Results indicated that islanding affected the $N$. confucianus fatness between the sexes and the extent of their differences ranged from significant level (large islands, $P=0.015$ ) to no significance (small and medium islands, $P=0.063$ and 0.288); among age groups, only a few adjacent age groups had significant difference (large: $\hat{\delta}$, I and II, $P=0.042$; media: + and $\delta$ all II and III, $P=0.013$ and 0.044 ) or very significantly (large: + , III and IV, $P=0.000$ ), the others failed to meet the significant level $(P>0.05)$. The changes of $N$. confucianus fatness among age groups were mostly related to the changes of its own physiological characteristics; Isolated island resulted in significant difference in different types of dominant species and abundance, thus affecting the difference of fatness; There was significant difference $(P=0.000)$ between different sizes islands and had significant positive correlation between them (Pearson test, $r=0.561, P=0.037$ ). In a word, in addition to sex and age, the islands area, habitat and population pressure all have a direct or indirect relationship with the difference of fatness in Qiandao Lake, which also reflects the adaptability of $N$. confucianus to
\end{abstract}

收稿日期：2008-12-18; 接受日期：2009-08-18

基金项目：浙江省自然科学基金资助项目 (Y507080)

“通讯作者 (Corresponding author), E-mail: sky90@zjnu.cn

第一作者简介: 孙 波, 男, 硕士研究生, 主要从事动物生态学研究 
islanding environment.

Key words: Niviventer confucianus; Relative fatness; Habitat fragmentation; Island of Qiandao Lake

肥满度是一种表征动物生理或营养状况的形 态生理指数 (Xia \& Sun, 1963), 可以衡量动物身体 的状况, 在生态学及农业生产中都有应用（Badiani et al, 1997; Guinet et al, 1998; Stirling et al, 1999; Murray, 2002; Lloret \& Planes, 2003)。研究鼠类肥满 度, 能够从内因阐述鼠类对环境的适应, 对于分析 种群动态具有重要意义。Xia \& Sun (1963) 首次将 表示鱼类生活状况的肥满度指标引用到啮齿动物 生态学的研究领域, 随后众多学者对大林姬鼠 (Apodemus speciosus) (Xia \& Sun, 1964)、小家鼠 (Mus musculus) (Yan, 1983)、灰仓鼠 (Cricetulus migratorius) (Zhong \& Yan, 1984)、达乌尔黄鼠 (Spermophilus dauricus) (Liu et al, 1990)、大仓鼠 (Cricetulus triton) ( Li \& Wang, 1992)、黑线仓鼠 (Cricetulus barabensis) (Zhou et al, 1992)、黑线姬 鼠 (Apodemus agrarius) (Yang, 1995) 和社鼠 (Niviventer confucianus) (Gao \& Bao, 1995) 等鼠 类的肥满度进行了研究。

生境片段化是近年来生态学领域研究的热点 (Theobald et al, 1997; Laurance et al, 2002)。生境 片段化是通过影响生物个体的行为、种群动态进而 作用于生态系统的各个环节 (Debinski \& Holt, 2000; Zschokke et al, 2001; Fahrig, 2003)。岛屿隔离也是 生境片段化的一种 (Lu et al, 2005), 目前在此方面 的研究主要是岛屿隔离对生物多样性的影响

(Diamond, 2001; Terborgh et al, 2001; Wu et al, 2003)。Gao et al（1995）开展过舟山岛（海岛）与 金华北山 (内陆) 社鼠的肥满度之研究, 揭示了海 岛与内陆之间社鼠肥满度在性别、年龄、季节等方 面的差异, 但未涉及岛屿隔离状况下肥满度的特 征。那么, 在岛屿隔离状况下, 岛屿的特征等生态 因子是否对鼠类的肥满度产生影响? 为此, 本文选 取千岛湖这一典型的岛屿化生境, 对其优势鼠种一 一社鼠进行肥满度研究, 以探讨岛屿的隔离对社鼠 的肥满度之影响及其作用机理。

\section{1 研究地区概况与方法}

\section{1 研究地区概况}

千岛湖位于浙江省西部淳安县境内, 介于 $118^{\circ} 34^{\prime} \sim 119^{\circ} 15^{\prime} \mathrm{E}, 29^{\circ} 22^{\prime} \sim 29^{\circ} 50^{\prime} \mathrm{N}$ 之间, 东西长 60 $\mathrm{km}$, 南北宽 $50 \mathrm{~km}$, 正常水位 $108 \mathrm{~m}$ (黄海高程), 拥有 $573 \mathrm{~km}^{2}$ 水面和 $409 \mathrm{~km}^{2}$ 山场、178 亿 $\mathrm{m}^{3}$ 蓄水 量, 是 1959 年因建造新安江水电站筑坝蓄水而形 成的人工湖。千岛湖上星罗棋布的许多岛屿只是水 位上涨后未完全被水淹没的山峰, 因湖内有 2500 $\mathrm{m}^{2}$ 以上岛屿 1078 个而得名。千岛湖地处亚热带季 风气候区的北缘, 气候温暖湿润, 四季分明, 年平 均气温为 $17{ }^{\circ} \mathrm{C}$, 平均降水量 $1430 \mathrm{~mm}$, 年相对湿 度 76\%。千岛湖区域植被类型属亚热带常绿林, 湖 中岛屿植被以天然次生马尾松 (Pinus massoniana) 林为主。千岛湖是国家级风景名胜区, 也是中国目 前最大的森林公园。

\section{2 研究方法}

根据Terborgh et al (2001) 的研究方法结合研 究区域的实际情况, 对千岛湖的岛屿依据面积 $(\mathrm{S})$ 划分为 3 种类型: 大型岛屿 $\left(\mathrm{S}>30 \mathrm{hm}^{2}\right)$ 、中型岛 屿 $\left(2 \mathrm{hm}^{2}<\mathrm{S} \leqslant 30 \mathrm{hm}^{2}\right)$ 、小型岛屿 $\left(0.01 \mathrm{hm}^{2} \leqslant \mathrm{~S} \leqslant\right.$ $2 \mathrm{hm}^{2}$ ), 同时参考浙江省千岛湖 $1: 10000$ 地图, 以及走访当地农民确定取样岛屿。选取彼此隔离的 大型岛屿 5 个、中型岛屿 4 个和小型岛屿 5 个作为 研究样地, 研究样地的具体特征见表 1 。

2008 年 5 月在所选取的岛屿中, 采用夹夜法 (中 号铁板夹) 开展小型兽类种群数量调查, 对捕获的 社鼠进行编号, 记录所捕地点的生境类型, 鉴定性 别, 测量体重、体长、尾长等数据, 并且解剖观察 其繁殖状况。

以体重法（Bao \& Zhuge, 1984）划分社鼠的年 龄, 各年龄组的划分指标为: 体重 $\leqslant 35.0 \mathrm{~g}$ 为幼年 组 ( I ); $35.0 \mathrm{~g}<$ 体重 $\leqslant 50.0 \mathrm{~g}$ 为亚成年组 ( II ); $50.0 \mathrm{~g}<$ 体重 $\leqslant 80.0 \mathrm{~g}$ 为成年组 (III); 体重 $>80.0 \mathrm{~g}$ 为老年组 $(I V)$ 。

以 $K=100 \mathrm{~W} / \mathrm{L}^{3}$ 计算社鼠的肥满度 (Xia \& Sun, $1963)$, 式中 $K$ 为肥满度 $\left(\mathrm{g} / \mathrm{cm}^{3}\right), W$ 为体重 $(\mathrm{g}), L$ 为体长 $(\mathrm{cm})$ 。一般肥满度值越大, 表示鼠类身体 状况越好。

采用 $t$-检验分析性别、相邻年龄组肥满度差异; 用单因素方差 (one-way ANOVA) 检验分析生境间 以及不同面积岛屿内肥满度差异; 用 Pearson 相关 检验分析各岛屿社鼠种群相对数量 (捕获率) 与肥 满度的关系。 
所涉及的数据处理及差异性分析均在统计软 件 SPSS11.5 中进行, $P \leqslant 0.05$ 为显著差异, $P \leqslant 0.01$ 为极显著差异, $P>0.05$ 为差异不显著。

\section{2 结 果}

根据岛屿的面积大小确定置夹数量, 每个大型 岛置夹 900 只, 中型岛置夹 600 只, 小型岛置夹 300 只。14 个取样岛屿, 共置鼠夹 8400 只, 捕获社鼠 标本 648 只, 其中雌鼠 363 只 (均未怀孕), 雄鼠 285 只 (表 2)。

表 1 取样岛屿特征

Tab. 1 Characteristics on each island

\begin{tabular}{|c|c|c|c|c|}
\hline 样地位置 Sample & 地理坐标 Geographic coordinate & 岛屿面积 Area $\left(\mathrm{hm}^{2}\right)$ & 岛屿等级 Class & 植被类型 Cover type \\
\hline 界首岛 Jieshou & $\mathrm{N}: 29^{\circ} 30^{\prime} 32.6^{\prime \prime}$ E: $118^{\circ} 52^{\prime} 49.8^{\prime \prime}$ & 1289 & $\mathrm{~L}$ & Cbmf \\
\hline 大畈龙 Dafanlong & $\mathrm{N}: 29^{\circ} 31^{\prime} 04.9^{\prime \prime}$ E: $118^{\circ} 55^{\prime} 10.4^{\prime \prime}$ & 109.03 & $\mathrm{~L}$ & Cbmf \\
\hline 高仙阁 Gaoxiange & $\mathrm{N}: 29^{\circ} 31^{\prime} 37.3^{\prime \prime}$ E: $118^{\circ} 56^{\prime} 04.6^{\prime \prime}$ & 55.08 & $\mathrm{~L}$ & $\mathrm{Cf}$ \\
\hline 乌石岛 Wushi & $\mathrm{N}: 29^{\circ} 29^{\prime} 30.7^{\prime \prime}$ E: $118^{\circ} 53^{\prime} 56.9^{\prime \prime}$ & 46.37 & $\mathrm{~L}$ & $\mathrm{Cf} 、 \mathrm{Cbmf}$ \\
\hline 东门岛 Dongmen & $\mathrm{N}: 29^{\circ} 32^{\prime} 05.8^{\prime \prime}$ E: $118^{\circ} 55^{\prime} 47.9^{\prime \prime}$ & 32.29 & $\mathrm{~L}$ & $\mathrm{Cf} 、 \mathrm{Cbmf}$ \\
\hline 茶坞岭 II Chawuling II & $\mathrm{N}: 29^{\circ} 31^{\prime} 21.9^{\prime \prime} \mathrm{E}: 118^{\circ} 55^{\prime} 37.1^{\prime \prime}$ & 12.02 & M & $\mathrm{Cf} 、 \mathrm{Bf}$ \\
\hline 竹林坞 Zhulinwu & $\mathrm{N}: 29^{\circ} 29^{\prime} 31.4^{\prime \prime}$ E: $118^{\circ} 52^{\prime} 34.5^{\prime \prime}$ & 9.12 & M & $\mathrm{Cf}$ \\
\hline 中岛 Zhongdao & $\mathrm{N}: 29^{\circ} 30^{\prime} 07.7^{\prime \prime} \mathrm{E}: 118^{\circ} 53^{\prime} 19.2^{\prime \prime}$ & 2.9 & M & $\mathrm{Bf}$ \\
\hline 灯塔岛 I Dengta I & $\mathrm{N}: 29^{\circ} 29^{\prime} 47.2^{\prime \prime} \quad \mathrm{E}: 118^{\circ} 54^{\prime} 28.2^{\prime \prime}$ & 2.83 & M & $\mathrm{Cf}$ \\
\hline 灯塔岛 II Dengta II & $\mathrm{N}: 29^{\circ} 29^{\prime} 52.2^{\prime \prime} \mathrm{E}: 118^{\circ} 54^{\prime} 17.3^{\prime \prime}$ & 1.54 & $\mathrm{~S}$ & $\mathrm{Cf}$ \\
\hline 茶坞岭 I Chawuling I & $\mathrm{N}: 29^{\circ} 31^{\prime} 26.3^{\prime \prime}$ E: $118^{\circ} 55^{\prime} 40.9^{\prime \prime}$ & 1.08 & $\mathrm{~S}$ & $\mathrm{Cf} 、 \mathrm{Bf}$ \\
\hline 拦网头 Lanwangtou & $\mathrm{N}: 29^{\circ} 29^{\prime} 31.4^{\prime \prime}$ E: $118^{\circ} 52^{\prime} 34.5^{\prime \prime}$ & 1.01 & $\mathrm{~S}$ & $\mathrm{Cf}$ \\
\hline 竹丝坞 Zhusiwu & $\mathrm{N}: 29^{\circ} 29^{\prime} 23.5^{\prime \prime} \quad \mathrm{E}: 118^{\circ} 54^{\prime} 12.8^{\prime \prime}$ & 0.97 & $\mathrm{~S}$ & $\mathrm{O}$ \\
\hline 桥坞坪 Qiaowuping & $\mathrm{N}: 29^{\circ} 29^{\prime} 18.7^{\prime \prime} \mathrm{E}: 118^{\circ} 52^{\prime} 34.2^{\prime \prime}$ & 0.86 & $\mathrm{~S}$ & $\mathrm{Cf} 、 \mathrm{Bf}$ \\
\hline
\end{tabular}

L: 大型岛 (large island); M: 中型岛 (moderate island); S: 小型岛 (small island); Cbmf: 针阔混交林 (coniferous-broadleaved mixed forest); Cf: 针叶林 (coniferous forest); Bf: 竹林 (bamboo forest); O: 桔园 (orangery)。

表 214 个岛屿的社鼠捕获概况

Tab.2 The capture profiles of Niviventer confucianus on 14 islands

\begin{tabular}{lccc}
\hline \multicolumn{1}{c}{ 岛屿 Island } & $\begin{array}{c}\text { 置夹数 (只) } \\
\text { Number of mousetrap }\end{array}$ & $\begin{array}{c}\text { 社鼠样本数 (只) } \\
\text { Number of Niviventer confucianus }\end{array}$ & $\begin{array}{c}\text { 捕获率 }(\%) \\
\text { Capture rate }\end{array}$ \\
\hline 界首岛 Jieshou & 900 & 67 & 7.44 \\
大畈龙 Dafanlong & 900 & 109 & 12.11 \\
高仙阁 Gaoxiange & 900 & 33 & 3.67 \\
乌石岛 Wushi & 900 & 101 & 11.22 \\
东门岛 Dongmen & 900 & 61 & 6.78 \\
茶坞岭 II Chawuling II & 600 & 57 & 9.50 \\
竹林坞 Zhulinwu & 600 & 30 & 5.00 \\
中岛 Zhongdao & 600 & 93 & 15.50 \\
灯塔岛 I Dengta I & 600 & 18 & 3.00 \\
灯塔岛 II Dengta II & 300 & 11 & 3.67 \\
茶坞岭 I Chawuling I & 300 & 17 & 5.67 \\
拦网头 Lanwangtou & 300 & 10 & 3.33 \\
竹丝坞 Zhusiwu & 300 & 25 & 8.33 \\
桥坞坪 Qiaowuping & 300 & 16 & 5.33 \\
\hline
\end{tabular}

\section{1 肥满度与性别的关系}

对不同性别的肥满度进行统计 (表 3), 结果显 示: 雌雄鼠间的肥满度变动幅度均较大, 雌鼠肥满 度平均值高于雄鼠, 两性间差异极显著 $(P=0.010)$ 。

\section{2 肥满度与年龄的关系}

分别比较雌雄鼠年龄组间的肥满度之差异 (表 4), 结果发现, 除雌鼠成年组 (III) 与老年组 (IV) 差异极显著外 $(P=0.001)$, 其余年龄组间差异均不
显著 $(P>0.05)$ 。这表明肥满度在年龄组间的变化不 明显。

\section{3 肥满度与生境的关系}

由表 1 可知, 岛屿上的生境总共有以下 4 种类 型：针阔混交林、针叶林、桔园、竹林。对捕获于 这 4 种类型生境中的个体进行肥满度分析（表 5)。

社鼠的肥满度在 4 种生境中差异极显著 $\left(F_{(3,644)}=6.326, P<0.01\right)$, 肥满度的均值以竹林最 
高, 其次为针阔混交林和桔园, 针叶林最低。对不 同性别来说，上述生境中雌鼠肥满度差异不显著
$\left(F_{(3,359)}=2.372, P>0.05\right)(n=363)$, 而雄鼠差异极 显著 $\left(F_{(3,281)}=5.296, P<0.01\right)(n=285)$ 。

表 3 社鼠肥满度的性别差异

Tab. 3 Sexual difference of the relative fatness of Niviventer confucianus

\begin{tabular}{|c|c|c|c|c|c|}
\hline 性别 Sex & $\begin{array}{l}\text { 样本数 } \\
\text { Number of sample }\end{array}$ & $\begin{array}{l}\text { 范围 } \\
\text { Range }\end{array}$ & $\begin{array}{l}\text { 平均值 } \pm \text { 标准误 } \\
\bar{X} \pm S E\end{array}$ & $\begin{array}{l}\text { 标准差 } \\
S D\end{array}$ & $\begin{array}{l}t \text { 检验 } \\
t \text {-test }\end{array}$ \\
\hline 雌性 Female (q) & 363 & $1.53 \sim 5.05$ & $2.78 \pm 0.37$ & 0.52 & $t=2.598$ \\
\hline 雄性 Male ( $\left.{ }^{\circledR}\right)$ & 285 & $1.35 \sim 4.58$ & $2.67 \pm 0.36$ & 0.46 & $P=0.010^{* *}$ \\
\hline
\end{tabular}

表 4 社鼠不同年龄组肥满度的变化

Tab. 4 Variation on the relative fatness of Niviventer confucianus in different age groups

\begin{tabular}{|c|c|c|c|c|c|c|}
\hline \multirow[b]{2}{*}{$\begin{array}{c}\text { 年龄组 } \\
\text { Age group }\end{array}$} & \multicolumn{3}{|c|}{ 雌 Female (q) } & \multicolumn{3}{|c|}{ 雄 Male ( ঐ) } \\
\hline & $\begin{array}{c}\text { 样本数 } \\
\text { Number } \\
\text { of sample }\end{array}$ & $\begin{array}{c}\text { 平均值 } \pm \text { 标准误 } \\
\bar{X} \pm S E\end{array}$ & $t$ 检验 $t$-text & $\begin{array}{c}\text { 样本数 } \\
\text { Number } \\
\text { of sample }\end{array}$ & $\begin{array}{c}\text { 平均值 } \pm \text { 标准误 } \\
\bar{X} \pm S E\end{array}$ & $t$ 检验 $t$-test \\
\hline I & 40 & $(2.93 \pm 0.10)$ & $t=1.554, P=0.122$ & 22 & $2.58 \pm 0.75$ & $t=1.730, P=0.088$ \\
\hline \multirow[t]{2}{*}{ II } & 115 & $(2.72 \pm 0.45)$ & & 54 & $2.39 \pm 0.11$ & \\
\hline & & & $t=0.747, P=0.456$ & & & $t=1.847, P=0.066$ \\
\hline III & 195 & $(2.77 \pm 0.67)$ & & 169 & $2.71 \pm 0.46$ & \\
\hline IV & 13 & $(3.16 \pm 0.16)$ & $t=3.261, P=0.001^{* *}$ & 40 & $2.81 \pm 1.03$ & $t=1.328, P=0.186$ \\
\hline
\end{tabular}

表 5 不同生境社鼠肥满度的变化

Tab. 5 Variation on the relative fatness of Niviventer confucianus in different habitats

\begin{tabular}{|c|c|c|c|c|c|c|c|c|c|c|}
\hline \multirow{2}{*}{ 性别 } & \multirow{2}{*}{ Sex } & \multicolumn{2}{|c|}{$\begin{array}{c}\text { 针阔混交林 } \\
\text { Coniferous-broadleaved } \\
\text { Mixed Forest }\end{array}$} & \multicolumn{2}{|c|}{$\begin{array}{c}\text { 针叶林 } \\
\text { Coniferous forest }\end{array}$} & \multicolumn{2}{|c|}{$\begin{array}{c}\text { 桔园 } \\
\text { Orangery }\end{array}$} & \multicolumn{2}{|c|}{$\begin{array}{c}\text { 竹林 } \\
\text { Bamboo forest }\end{array}$} & \multirow{2}{*}{$\begin{array}{c}\text { 生境差异 } \\
\text { Difference } \\
\text { between } \\
\text { habitats }\end{array}$} \\
\hline & & $\begin{array}{l}\text { 样本数 } \\
\text { Number } \\
\text { of sample }\end{array}$ & $\begin{array}{l}\text { 平均值 } \\
\pm \text { 标准误 } \\
\bar{X} \pm S E\end{array}$ & $\begin{array}{l}\text { 样本数 } \\
\text { Number } \\
\text { of sample }\end{array}$ & $\begin{array}{l}\text { 平均值 } \\
\pm \text { 标准误 } \\
\bar{X} \pm S E\end{array}$ & $\begin{array}{l}\text { 样本数 } \\
\text { Number } \\
\text { of sample }\end{array}$ & $\begin{array}{l}\text { 平均值 } \\
\pm \text { 标准误 } \\
\bar{X} \pm S E\end{array}$ & $\begin{array}{l}\text { 样本数 } \\
\text { Number } \\
\text { of sample }\end{array}$ & $\begin{array}{l}\text { 平均值 } \\
\pm \text { 标准误 } \\
\bar{X} \pm S E\end{array}$ & \\
\hline 우 & & 111 & $2.81 \pm 0.70$ & 149 & $2.70 \pm 0.19$ & 12 & $2.65 \pm 0.11$ & 91 & $(2.87 \pm 0.72)$ & $\begin{array}{c}F_{(3,359)}=2.372 \\
P=0.070\end{array}$ \\
\hline$\delta$ & & 95 & $2.72 \pm 0.56$ & 117 & $2.55 \pm 0.14$ & 13 & $2.81 \pm 0.11$ & 60 & $(2.80 \pm 0.77)$ & $\begin{array}{c}F_{(3,281)}=5.296 \\
P=0.001^{* *}\end{array}$ \\
\hline $\begin{array}{l}\text { 总 } \\
\text { Total }\end{array}$ & 计 & 206 & $2.77 \pm 0.50$ & 266 & $2.64 \pm 0.42$ & 25 & $2.73 \pm 1.08$ & 151 & $(2.84 \pm 0.53)$ & $\begin{array}{c}F_{(3,644)}=6.326 \\
P=0.000^{* *}\end{array}$ \\
\hline
\end{tabular}

**差异极显著, $P \leqslant 0.01$ 。 ${ }^{* *}$ highly significant differences, $P \leqslant 0.01$.

\section{4 肥满度与岛屿类型的关系}

2.4.1 肥满度与岛屿面积 对 14 个不同岛屿的社 鼠的肥满度进行统计分析 (表 6), 显示不同岛屿间 社鼠肥满度差异极显著 $\left(F_{(13,634)}=2.923, P<0.01\right)$, 且肥满度的均值与岛屿面积成显著正相关 (Pearson test, $r=0.561, P<0.05$ ), 表明岛屿大小可以对社鼠 肥满度的变化产生一定的影响, 即随着岛屿面积的 增减, 社鼠的肥满度也呈现高低变化。

2.4.2 不同类型岛屿与肥满度的性别差异 不同 类型岛屿中的雌雄鼠的肥满度间性别差异 (表 7)。
从表中可以看出, 岛屿类型不同, 雌雄鼠肥满度的 变化规律也不相同。就肥满度变化范围而言, 中型 岛屿相对较高, 雌雄变幅分别为 3.25 和 3.01 , 其次 为大型岛屿 $(9,3.18 ; \widehat{O}, 2.45)$, 小型岛屿则相 对较低 ( $9,2.29 ; \hat{O}, 1.95)$; 就肥满度均值来看, 大中型岛屿为䧳性高于雄性, 小型岛屿为雄性高于 雌性; 而进行两性间的肥满度差异的比较, 发现除 大型岛屿差异显著外 $(P=0.015)$, 其他皆不显著 $(P>0.05)$ 。

2.4.3 不同类型岛屿与肥满度的年龄差异 表 8 的 
表 6 肥满度与岛屿面积的关系

Tab. 6 Relation between the relative fatness and the area of island

\begin{tabular}{|c|c|c|c|c|c|c|}
\hline \multirow[b]{2}{*}{ 岛屿 Island } & \multirow{2}{*}{$\begin{array}{l}\text { 岛屿面积 } \\
\text { Area（ha） }\end{array}$} & \multicolumn{4}{|c|}{ 肥满度值 The value of fatness indexes } & \multirow{2}{*}{$\begin{array}{c}\text { One-way ANOVA } \\
\text { 检验 }\end{array}$} \\
\hline & & $\begin{array}{c}\text { 样本数 } \\
\text { Number of sample }\end{array}$ & $\begin{array}{l}\text { 范围 } \\
\text { Range }\end{array}$ & $\begin{array}{c}\text { 平均值士标准误 } \\
\bar{X} \pm S E\end{array}$ & $\begin{array}{c}\text { 标准差 } \\
S D\end{array}$ & \\
\hline 界首岛 Jieshou & 1289 & 67 & $1.93-4.71$ & $2.97 \pm 0.92$ & 0.55 & \\
\hline 大畈龙 Dafanlong & 109.03 & 109 & $1.89-4.14$ & $2.63 \pm 0.49$ & 0.38 & \\
\hline 高仙阁 Gaoxiange & 55.08 & 33 & $2.27-3.68$ & $2.92 \pm 0.94$ & 0.40 & \\
\hline 乌石岛 Wushi & 46.37 & 101 & $1.44-3.92$ & $2.62 \pm 0.65$ & 0.48 & \\
\hline 东门岛 Dongmen & 32.29 & 61 & $1.40-4.17$ & $2.63 \pm 0.81$ & 0.47 & \\
\hline 茶坞岭 II Chawuling II & 12.02 & 57 & $1.35-4.32$ & $2.77 \pm 0.90$ & 0.50 & \\
\hline 竹林坞 Zhulinwu & 9.12 & 30 & $2.07-3.90$ & $2.80 \pm 1.26$ & 0.51 & $F_{(13,634)}=2.923$, \\
\hline 中岛 Zhongdao & 2.9 & 93 & $2.04-5.05$ & $2.78 \pm 0.72$ & 0.51 & $P=0.000^{* *}$ \\
\hline 灯塔岛 I Dengta I & 2.83 & 18 & $1.67-4.96$ & $2.80 \pm 0.18$ & 0.78 & \\
\hline 灯塔岛 II Dengta II & 1.54 & 11 & $2.42-3.97$ & $2.76 \pm 0.14$ & 0.46 & \\
\hline 茶坞岭 I Chawuling I & 1.08 & 17 & $2.10-3.28$ & $2.68 \pm 1.15$ & 0.35 & \\
\hline 拦网头 Lanwangtou & 1.01 & 10 & $2.29-4.22$ & $2.72 \pm 0.19$ & 0.60 & \\
\hline 竹丝坞 Zhusiwu & 0.97 & 25 & $2.00-3.89$ & $2.74 \pm 1.08$ & 0.40 & \\
\hline 桥坞坪 Qiaowuping & 0.86 & 16 & $1.93-4.05$ & $2.56 \pm 0.13$ & 0.52 & \\
\hline
\end{tabular}

${ }^{* *}$ 差异极显著, $P \leqslant 0.01$ 。 ${ }^{* *}$ highly significant differences, $P \leqslant 0.01$.

表 7 不同类型岛屿社鼠肥满度的性别差异

Tab.7 Sexual difference of the relative fatness of Niviventer confucianus in different type islands

\begin{tabular}{|c|c|c|c|c|c|c|}
\hline 岛屿类型 Type island & 性别 Sex & $\begin{array}{c}\text { 样本数 } \\
\text { Number of sample }\end{array}$ & 范围 Range & $\begin{array}{c}\text { 平均值 } \pm \text { 标准误 } \\
\bar{X} \pm S E\end{array}$ & $\begin{array}{c}\text { 标准差 } \\
S D\end{array}$ & $\begin{array}{l}t \text { 检验 } \\
t \text {-test }\end{array}$ \\
\hline \multirow{2}{*}{ 大型岛屿 Large island } & 웅 & 202 & $1.53-4.71$ & $2.77 \pm 0.48$ & 0.51 & \multirow{2}{*}{$t=2.448, P=0.015^{*}$} \\
\hline & $\hat{\sigma}$ & 169 & $1.40-3.85$ & $2.65 \pm 0.46$ & 0.44 & \\
\hline \multirow{2}{*}{ 中型岛屿 Moderate island } & 운 & 114 & $2.04-5.05$ & $2.84 \pm 0.72$ & 0.56 & \multirow{2}{*}{$t=1.873, P=0.063$} \\
\hline & $\widehat{\partial}$ & 84 & $1.35-4.58$ & $2.70 \pm 0.71$ & 0.48 & \\
\hline \multirow{2}{*}{ 小型岛屿 Small island } & 운 & 47 & $1.93-4.22$ & $2.65 \pm 0.83$ & 0.42 & \multirow{2}{*}{$t=1.070, P=0.288$} \\
\hline & $\hat{\sigma}$ & 32 & $2.10-4.05$ & $2.76 \pm 1.14$ & 0.47 & \\
\hline
\end{tabular}

"表示差异显著, $P \leqslant 0.05$ 。 ${ }^{*}$ significant differences, $P \leqslant 0.05$.

数据表明, 在所比较的 18 组数据中, 仅大、中型 岛屿中各有 2 个相邻年龄组肥满度差异显著 $(P<0.05$ ) (大型岛屿: $\delta$, I 与 II, $P=0.042$; 中型 岛屿: $P$ 和 $\gamma$ 皆为 II 与 III, $P=0.013$ 和 $P=0.044$ ) 或 极显著 $(P<0.01)$ (大型岛屿: + , III 与 IV, $P=0.000$ ), 仅占所比较数据的 $22.2 \%$, 其余相邻年龄组间均未 达到显著水平 $(P>0.05)$, 可见, 岛屿类型的变化同 样未引起社鼠的肥满度年龄组间的显著差异。

\section{5 肥满度与各岛屿种群数量的关系}

捕获率可以显示种群的相对数量。对捕获率所 示的各岛屿该鼠种群的相对数量与其肥满度进行 Pearson 相关检验 (为避免雌雄鼠的行为差异带来的 影响, 对雌雄鼠分别进行分析) 的结果显示 (表 9): 各岛屿捕获率与雌鼠肥满度的相关系数为 $r=-0.226$

$(P=0.437)$, 与雄鼠相关系数为 $r=-0.019$ $(P=0.949)$, 捕获率与肥满度均无显著相关性 $(P>0.05)$ 。

\section{3 讨 论}

栖息地岛屿的面积、形状、隔绝度等对岛屿生 物群落具有重要的影响, 使岛屿生物群落特征产生 重大的变化 (Robinson et al, 1992; Wiggins \& Moller, 1997)。生境片段化不但会直接导致动植物栖息地 环境被破坏，生物多样性下降（Wadsworth \& Swetnam, 1988; Metzger, 2000), 还会影响动植物种 群动态从而改变群落组成 (Holt et al, 1995; Harrison, 1999); 并且片段后产生边缘效应, 通过引起生物 和非生物条件的变化, 包括食物、栖息地、繁殖场 所等, 进而影响斑块中的有机体 (Murcia, 1995; Gehlhausen et al, 2000); 同时，生境片段化也导致 了原生境的总面积减小, 产生隔离的异质种群, 影 响到个体的行为特性 (Saunders et al, 1991; Davies \& Margules, 1998)。 
表 8 不同类型岛屿社鼠肥满度的年龄差异

Tab. 8 Age difference of the relative fatness of Niviventer confucianus in different type islands

\begin{tabular}{|c|c|c|c|c|c|c|c|}
\hline \multirow[b]{2}{*}{$\begin{array}{l}\text { 岛屿类型 } \\
\text { Type island }\end{array}$} & \multirow[b]{2}{*}{$\begin{array}{c}\text { 年龄组 } \\
\text { Age groups }\end{array}$} & \multicolumn{3}{|c|}{ 雌 Female (q) } & \multicolumn{3}{|c|}{ 雄 Male（ふ⿱亠乂 } \\
\hline & & $\begin{array}{c}\text { 样本数 } \\
\text { Number } \\
\text { of sample }\end{array}$ & $\begin{array}{c}\text { 平均值 } \pm \text { 标准误 } \\
\bar{X} \pm S E\end{array}$ & $\begin{array}{l}t \text { 检验 } \\
t \text {-text }\end{array}$ & $\begin{array}{c}\text { 样本数 } \\
\text { Number } \\
\text { of sample }\end{array}$ & $\begin{array}{c}\text { 平均值士标准误 } \\
\bar{X} \pm S E\end{array}$ & $\begin{array}{c}t \text { 检验 } \\
t \text {-test }\end{array}$ \\
\hline \multirow{5}{*}{ 大型岛屿 L } & I & 19 & $2.90 \pm 0.14$ & \multirow{2}{*}{$t=1.811, P=0.074$} & 13 & $2.63 \pm 1.04$ & \multirow[t]{2}{*}{$t=2.099, P=0.042^{*}$} \\
\hline & II & 64 & $2.67 \pm 0.77$ & & 32 & $2.30 \pm 0.16$ & \\
\hline & & & & \multirow[t]{2}{*}{$t=1.251, P=0.213$} & & & \multirow[t]{2}{*}{$t=0.473, P=0.637$} \\
\hline & III & 113 & $2.76 \pm 0.61$ & & 104 & $2.67 \pm 0.52$ & \\
\hline & IV & 6 & $3.53 \pm 0.26$ & $t=3.803, P=0.000^{* *}$ & 20 & $2.80 \pm 0.11$ & $t=1.282, P=0.202$ \\
\hline \multirow{5}{*}{ 中型岛屿 M } & I & 17 & $2.95 \pm 0.16$ & \multirow[t]{2}{*}{$t=0.131, P=0.896$} & 9 & $2.52 \pm 0.13$ & \multirow[t]{2}{*}{$t=0.224, P=0.825$} \\
\hline & II & 40 & $2.69 \pm 0.87$ & & 16 & $2.48 \pm 0.10$ & \\
\hline & & & & \multirow[t]{2}{*}{$t=2.525, P=0.013^{*}$} & & & \multirow[t]{2}{*}{$t=2.056, P=0.044^{*}$} \\
\hline & III & 52 & $2.86 \pm 0.11$ & & 47 & $2.77 \pm 1.00$ & \\
\hline & IV & 5 & $2.98 \pm 1.35$ & $t=0.795, P=0.430$ & 12 & $2.81 \pm 0.14$ & $t=0.237, P=0.814$ \\
\hline \multirow{5}{*}{ 小型岛屿 S } & I & 4 & $2.93 \pm 0.44$ & \multirow[t]{2}{*}{$t=1.287, P=0.221$} & - & - & \\
\hline & II & 11 & $2.55 \pm 1.27$ & & 6 & $2.59 \pm 1.22$ & \\
\hline & & & & \multirow[t]{2}{*}{$t=0.611, P=0.545$} & & & \multirow[t]{2}{*}{$t=0.785, P=0.441$} \\
\hline & III & 30 & $2.63 \pm 0.96$ & & 18 & $2.77 \pm 0.12$ & \\
\hline & IV & 2 & $2.80 \pm 0.26$ & $t=0.572, P=0.572$ & 8 & $2.86 \pm 0.19$ & $t=0.403, P=0.691$ \\
\hline
\end{tabular}

“差异显著, $P \leqslant 0.05$ 。 "significant differences, $P \leq 0.05$.

表 9 社鼠肥满度与捕获率之间关系

Tab. 9 Relation between the relative fatness and the capture rate of Niviventer confucianus

\begin{tabular}{|c|c|c|c|}
\hline \multirow{2}{*}{ 样地 Sample } & \multirow{2}{*}{ 捕获率(C) Capture rate (\%) } & \multicolumn{2}{|c|}{ 肥满度值 The value of relative fatness indexes } \\
\hline & & q & $\partial$ \\
\hline 界首岛 Jieshou & 7.44 & $3.08 \pm 0.12$ & $2.87 \pm 0.96$ \\
\hline 大畈龙 Dafanlong & 12.11 & $2.64 \pm 0.67$ & $2.62 \pm 0.72$ \\
\hline 高仙阁 Gaoxiange & 3.67 & $3.01 \pm 1.04$ & $2.72 \pm 0.12$ \\
\hline 乌石岛 Wushi & 11.22 & $2.67 \pm 0.91$ & $2.55 \pm 0.90$ \\
\hline 东门岛 Dongmen & 6.78 & $2.72 \pm 1.09$ & $2.55 \pm 1.17$ \\
\hline 茶坞岭 II Chawuling II & 9.50 & $2.84 \pm 1.22$ & $2.68 \pm 1.32$ \\
\hline 竹林坞 Zhulinwu & 5.00 & $2.90 \pm 0.14$ & $2.66 \pm 0.11$ \\
\hline 中岛（hongdao & 15.50 & $2.80 \pm 0.97$ & $2.73 \pm 1.03$ \\
\hline 灯塔岛 I Dengta I & 3.00 & $3.07 \pm 0.39$ & $2.66 \pm 0.20$ \\
\hline 灯塔岛 II Dengta II & 3.67 & $2.69 \pm 1.27$ & $2.89 \pm 0.37$ \\
\hline 茶坞岭 I Chawuling I & 5.67 & $2.75 \pm 1.22$ & $2.52 \pm 0.19$ \\
\hline 拦网头 Lanwangtou & 3.33 & $2.84 \pm 0.26$ & $2.45 \pm 0.85$ \\
\hline 竹丝坞 Zhusiwu & 8.33 & $2.65 \pm 0.11$ & $2.81 \pm 0.11$ \\
\hline 桥坞坪 Qiaowuping & 5.33 & $2.33 \pm 0.110$ & $2.87 \pm 0.21$ \\
\hline $\begin{array}{l}\text { Pearson 相关检验 } \\
\text { Pearson test }\end{array}$ & $\begin{array}{l}\mathbf{C} \text { 和 } \phi \\
\mathbf{C} \text { 和 } \\
\end{array}$ & $r=-0.226, \quad n=14, \quad P=0.437$ & $r=-0.019, \quad n=14, \quad P=0.949$ \\
\hline
\end{tabular}

\section{1 岛屿化对社鼠的肥满度性别、年龄差异的影响} 在千岛湖岛屿, 从总体上看, 社鼠的肥满度均 值都是雌鼠高于雄鼠, 且两性间差异达到极显著水 平, 这种变化情况与同纬度浙江临安、金华北山及 舟山岛社鼠肥满度的变化无异 (Gao \& Bao, 1996)。 但在不同类型的岛屿中, 两性间的肥满度的变化规 律不同。大、中型岛屿中, 肥满度均值为雌鼠大于 雄鼠; 而小型岛屿中, 却为雄鼠大于雌鼠; 两性间
的肥满度之差异程度也由大型岛屿的显著差异 $(P<0.05)$ 至中小型岛屿的无显著差异（ $P>0.05 ）$ 变化。显然, 生境片段化在一定程度上对千岛湖岛 屿社鼠两性间的肥满度差异产生了影响, 即随片段 化程度的增高 (岛屿面积的减小), 社鼠活动、取 食范围受到限制的程度逐渐增加, 同一类型岛屿内 雌雄鼠对于能量消耗的差别变小, 肥满度两性间的 差异逐渐不明显。 
然而, 对千岛湖地区社鼠年龄组间的肥满度进 行总体和按岛屿类型统计后发现, 两种情况下, 肥 满度均值变化规律相似, 皆为幼年组高于亚成年 组, 即从亚成年组开始, 随年龄的增长而增长, 但 除个别相邻年龄组间出现显著 $(P<0.05)$ 或极显著 $(P>0.01)$ 差异外, 其余年龄组间均未达到显著水 平 $(P>0.05)$ 。这说明了岛屿化的因素未对社鼠的肥 满度在年龄上的差异产生显著影响。生理生态学研 究表明, 影响啮齿动物的个体大小及年龄可以通过 影响其基础代谢率（basal metabolic rate, BMR) 来 影响代谢率, 而个体大小便是决定 BMR 最主要的 因素 (McNab, 1980), 一般情况下, 代谢活性器官 的重量越大, BMR 就越大。小体重动物体内代谢 活性器官的重量相对较大, 因而单位质量的 BMR 较大 (Song \& Wang, 2002)。显然, 为满足高消耗 的基础代谢率, 幼年社鼠要具有较高的肥满度, 对 其存活有利, 且幼鼠受到亲鼠的抚育和照料, 营养 条件和生活环境相对优于其他年龄阶段, 所以肥满 度相对要高一些。动物进入亚成年阶段, 身体生长 发育明显加快, 生存难度加大, 因此, 肥满度较幼 鼠下降; 当动物长到成体以后, 体长基本上不再改 变, 而体重会因生境条件、季节等因素的变化发生 改变。Jenssen et al（1995）曾指出：动物在不同生 活史阶段用于生长、繁殖及生存 (包括越冬) 的能 量资源分配存在差异, 这种差异能导致个体大小和 其他形态、生理特征的性别和年龄差异。5 月份为 南方地区社鼠的繁殖高峰期 (Bao \& Zhuge, 1987), 鼠类参与繁殖哺育的过程, 是最耗能量的过程

(Mendl, 1988; Koivula et al, 2003), 如布氏田鼠 (Microtus brandti) 哺育期代谢能摄入最高时达正 常雌鼠的 323\%（Liu et al, 2003)。成年组和老年组 个体为满足哺育期需要高能量的消耗, 需要保证的 高肥满度, 会明显高于不参与繁殖的其他年龄组个 体。可见, 社鼠的肥满度年龄组间的变化主要与其 自身生理特征变化有关, 与岛屿化关系不明显。

\section{参考文献:}

Badiani A, Stipa S, Nanni N, Gatta PP, Manfredini M. 1997. Physical indices, processing yields, compositional parameters and fatty acid profile of three species of cultured Sturgeon (Genus Acipenser) [J]. Sci Food Agri, 74: 257-264.

Bao YX, Zhuge Y. 1984. The age estimation and age structure in the population and age structure in the population of Rattus niviventer confucianus [J]. Acta Theriol Sin, 4(2): 127-137. [鲍毅新, 诸葛阳. 1984. 社鼠的年龄鉴定与种群年龄组成. 兽类学报, 4(2): 127-137.]

\section{2 岛屿化的生境差异对社鼠的肥满度之影响}

由于岛屿的隔离隔作用, 不同类型岛屿上的植 被优势种及丰富度存在明显的差别 ( $\mathrm{Lu}$ et $\mathrm{al}$, 2005 ), 这种植被差异导致社鼠的肥满度产生差异。 社鼠是以植物的果实、种子以及根茎为主食 (Bao, 1993 ), 在所研究的 4 种植被类型中, 从食物资源 和隐蔽条件来看, 理应是针阔混交林最佳, 针叶林 次之, 竹林和桔园最差。但社鼠的肥满度却以竹林 最高, 其次为针阔混交林和桔园, 针叶林最低, 在 4 种生境中存在极显著差异。因本次研究中未开展 社鼠的食物丰富度调查, 因此这种差异的产生, 是 否与各生境地表及地下的食物可利用性相关还有 待于进一步的研究。

\section{3 岛屿化下, 社鼠的肥满度与种群数量的关系}

鼠类种群数量的变化和肥满度的关系可能受 到食物、栖息场所等影响（Liu et al, 2003), 因而, 种群相对数量低时, 其肥满度较高, 而种群数量高 时, 个体间竞争压力加剧, 导致其较差的生理状态, 肥满度就显示出较低数值。本文研究发现, 各岛捕 获率所示的社鼠种群的相对数量与其肥满度的 Pearson 相关检验均无显著相关性（ $P>0.05)$, 说明 了岛屿中社鼠的肥满度对其种群数量不产生影响。 究其原因可知, 岛屿环境中, 啮齿类动物的天敌大 大减少（Terborgh et al, 2001）, 其活动面积及取食 范围也就相对陆地种群增大, 加之社鼠个体适应能 力相对较强, 可取食物种类繁杂 (Bao, 1993), 因 而个体之间享有充足的空间资源和食物资源, 种群 压力产生的影响较小, 对肥满度的影响亦小。

总之, 在有近 50 年历史的千岛湖岛屿上, 社 鼠的肥满度在不同性别、年龄和不同类型岛屿等方 面产生了或多或少的差异，有些差异达到了显著或 极显著的水平。这些差异的产生，除了性别、年龄 等因素外, 与岛屿的面积、生境及种群压力有直接 或间接的关系，从侧面反映了社鼠也对岛屿环境的 适应性。

Bao YX, Zhuge Y. 1987. Ecological study of rodents in Jinhua Beishan mountain. Acta Theriol Sin, 7(4): 266-274. [鲍毅新，诸葛阳. 1987. 金 华北山啮齿类的生态研究. 兽类学报, 7(4): 266-274.]

Bao YX. 1993. A Summary of studies on Rattus niviventer [J]. J Zhejiang Norm Univ : Nat Sci, 16(2): 50-54. [鲍毅新. 1993. 社鼠的研究概要. 浙江师范大学学报: 自然科学版, 16(2): 50-54.]

Debinski DM, Holt RD. 2000. A survey and overview of habitat fragmentation experiments [J]. Conserv Biol, 14(2): 342-355. 
Diamond JM. 2001. Dammed experiments [J]. Science, 294: 1847-1848.

Davies KF, Margules CR. 1998. Effects of habitat fragmentation on carabid beetles: Experimental evidence [J]. J Anim Ecol, 67(3): 460-471.

Fahrig L. 2003. Effects of habitat fragmentation on biodiversity [J]. Аnnu Rev Ecol Syst, 34: 487-515.

Gehlhausen SM, Schwartz MW, Augspurger CK. 2000. Vegetation and microclimatic edge effects in two mixed-mesophytic forest fragments [J]. Plant Ecol, 147(1):2 1-35.

Guinet C, Roux JP, Bonnet M, Mison V. 1998. Effect of body size, body mass, and body condition on reproduction of female South African furseals (Arctocephalus pusillus) in Namibia [J]. Can J Zool, 76: 1418-1424.

Gao F, Bao YX. 1995. The comparison and analysis of relative fatness Rattus niviventer between Zhoushan island and Jinhua mountain [J]. Acta Theriol Sin, 15(4): 309. [高 枫, 鲍毅新. 1995. 舟山岛与金华 北山社鼠肥满度的比较与分析. 兽类学报, 15(4): 309.]

Gao F, Bao YX. 1996. A Study on the relative fatness of R. niviventer [J]. $J$ Zhejiang Norm Univ : Nat Sci, 19(1): 53-56. [高 枫, 鲍毅新. 1996. 社鼠肥满度的研究. 浙江师范大学学报: 自然科学版, 19(1): 53-56.]

Holt RD, Robinson GR, Gaines MS. 1995. Vegetation dynamics in an experimentally fragmented landscape [J]. Ecology, 76(5): 1610-1624.

Harrison S. 1999. Local and regional diversity in a patchy landscape native, alien, and endemic herbs on serpentine [J]. Ecology, 80(1): 70-80.

Jenssen TA, Congdon JD, Fischer RU, Estes R, Kling D, Edmands S. 1995. Morphological characteristics of the lizard Anolis Carolinensis from south Carolina [J]. Herpetologica, 51: 401-411.

Koivula M, Koskela E, Mappea T, Oksanen TA. 2003. Cost of reproduction in the wild: Manipulation of reproductive effort in the bank vole [J]. Ecology, 84 (2): 398-405.

Lloret J, Planes S. 2003. Condition, feeding and reproductive potential of white seabream Diplodus sargus as indicators of habitat quality and the effect of reserve protection in the northwestern Mediterranean [J]. Mar Ecol Prog Ser, 248: 197-208.

Laurance WF, Lovejoy TE, Vasconcelos HL, Bruna, EM, Didham RK, Stouffer PC, Gascon C, Bierregaard RO, Laurance SG., Sampaio E. 2002. Ecosystem decay of Amazonian forest fragments: A 22-year investigation [J]. Conserv Biol, 16: 605-618.

Liu ZC, Wang CG, Wang C, Ma KJ, Sun JF, Meng FY, Wang XJ. 1990. On the relative fatness of Spermophilus dauricus[J]. Acta Theriol Sin,10(1): 66-77. 刘振才, 王成贵, 王 琛, 马克俭, 孙景发, 孟繁玉, 王孝军. 1990. 达乌尔黄鼠 (Spermophilus dauricus) 肥满度的研究. 兽类学 报, 10 (1): 66-77.]

Li XC, Wang YZ. 1992. Studies on the relative fatness of greater long-tailed hamster [J]. Acta Theriol Sin, 12(4): 275-279. [李晓晨, 王廷正. 1992. 大仓鼠肥满度的研究. 兽类学报, 12 (4): 275-279.]

Lu JB, Ding LZ, Xu GF. 2005. Effects of islanding on plant species diversity in Thousand-island Lake region [J]. Chn J Appl Ecol, 16(9): 1672-1676. [卢剑波, 丁立仲, 徐高福. 2005. 千岛湖岛屿化对植物 多样性的影响初探. 应用生态学报, 16(9): 1672-1676.]

Liu H, Wang DH, Wang ZW. 2003. Energy requirements during reproduction in female brandt's voles [J]. J Mammal, 84 (4): 1410-1416.

Liu W, Yuan XR, Wang GH, Zhong WQ, Liu WD. 2003. Characteristics of relative fatness in Mongolian gerbil (Meriones unguiculatus) in different ages and seasons [J]. Acta Theriol Sin, 23(2): 139-144. [刘 伟, 宛新荣, 王广和, 钟文勤, 刘文东. 2003. 长爪沙鼠肥满度的年 龄和季节特征.兽类学报, 23(2): 139-144.]

Murray DL. 2002. Differential body condition and vulnerability to predation in snowshoe hares [J]. J Anim Ecol, 71: 614-625.

Metzger JP. 2000. Tree functional group richness and landscape structure in a Brazilian tropical fragmented landscape [J]. Ecol Appl, 10 (4): 1147-1161.

Murcia C. 1995. Edge effects in fragmented forests: implications for conservation [J]. Trends Ecol Evol Biol, 10(2): 58-62.

McNab BK. 1980. Food habits, energetics, and the population biology of mammals [J]. Am Nat, 116(1): 106-124.

Mendl M. 1988. The effects of litter size variation on mother-offspring relationships and behavioural and physical development in several mammalian species (principally rodents) [J]. J Zool Lond, 215(1): 15-34.

Robinson GR, Holt RD, Gaines MS, Hamburg SP, Johnson ML, Fitch HS, Martinko EA. 1992. Diverse and contrasting effects of habitat fragments [J]. Science, 257: 524-526.

Stirling I, Lunn NJ, Iacozza J. 1999. Long-term trends in the population ecology of polar bears in western Hudson Bay in relation to climatic change [J]. Arctic, 52(3): 294-306.

Saunders DA, Hobbs RJ, Margules CR. 1991. Biological consequences of ecosystem fragmentation: A review [J]. Conserv Biol, 5(1): 18-21.

Song ZG, Wang DH. 2002. Influencing factors on basal metabolic ratein mammals [J]. Acta Theriol Sin, 22(1): 54-60. [宋志刚, 王德华. 2002. 哺乳动物基础代谢率的主要影响因素. 兽类学报, 22(1): 54-60.]

Terborgh J, Lopez L, Nuñez P, Rao M, Shahabuddin G, Orihuela G, Riveros M, Ascanio R, Adler GH, Lambert TD, Balbas L. 2001. Ecological meltdown in predator-free forest fragments $[\mathrm{J}]$. Science, 294: 1923-1926.

Theobald DM, Miller JR, Hobbs NT. 1997. Estimating the cumulative effects of development on wildlife habitat [J]. Landscape Urban Plann, 39: 25-36.

Wu JG, Huang JH, Han XG, Xie ZQ, Gao XM. 2003. Three-gorges dam experiment in habitat fragmentation [J]. Science, 300: 1239-1240.

Wiggins DA, Moller AP. 1997. Island size, isolation, or inter specific competition? The breeding distribution of the Parus guild in the Danish archipelago [J]. Oecologia, 111: 255-260.

Wadsworth R, Swetnam R. 1988. Modelling the impact of climate warming at the landscape scale: will bench terraces become economically and ecologically viable structures under changed climates? [J]. Agri Ecosyst Environ, 68: 27-39.

Xia WP, Sun CL. 1963. Study on relative fatness of Clethrionomys rutilus Pallas [J]. Acta Zool Sin, 15 (1): 33-43. [夏武平, 孙崇潞. 1963. 红背 鼠肥巴满度的研究. 动物学报, 15 (1): 33-43.]

Xia WP, Sun CL. 1964. Study on relative fatness of Apodemus speciosus [J]. Acta Zool Sin, 16 (4): 555-565. [夏武平, 孙崇潞. 1964. 大林姬鼠肥 满度的研究. 动物学报, 16 (4): 555-565.]

Yan ZT. 1983. On the relative fatness of the house mice (Mus musculus L.) [J]. Acta Theriol Sin, 3 (2): 173-180. [严志堂. 1983. 小家鼠肥满度的 研究. 兽类学报, 3 (2): 173-180.]

Yang ZX. 1995. Study on the relative fatness of the striped field mouse (Apodemus agrarius) [J]. Acta Theriol Sin, 15 (1): 73-74. [杨再学. 1995. 黑线姬鼠肥满度的研究. 兽类学报, 15 (1): 73-74.]

Zhong MM, Yan ZT. 1984. On the relative fatness of the gray hamster (Cricetulus migratorius Pallas) [J]. Acta Theriol Sin, 4 (4): 273-282. [钟明明, 严志堂. 1984. 灰仓鼠 (Cricetulus migratorius Pallas) 肥满 度的研究. 兽类学报, 4 (4): 273-282.]

Zhou YL, Hou XX, Dong WH, Yang YP. 1992. A study on the relative fatness of striped hamser [J]. Acta Theriol Sin, 12(3): 207-212. [周延林, 侯希贤, 董维惠, 杨玉平. 1992. 黑线仓鼠肥满度的研究. 兽类学报, 12(3): 207-212.]

Zschokke S, Dolt C, Rusterholz HP. 2001 Short-term responses of plants and invertebrates to experimental small-scale grassland fragmentation [J]. Oecologia, 25(4): 559-572. 